\title{
Laser-Induced Hydrogen Radical Removal in UV MALDI- MS Allows for the Differentiation of Flavonoid Monoglycoside Isomers
}

\author{
Tohru Yamagaki, Takehiro Watanabe, Masaki Tanaka, Kohtaro Sugahara
}

Suntory Institute for Bioorganic Research, Shimamoto, Mishima, Osaka 618-0001, Japan

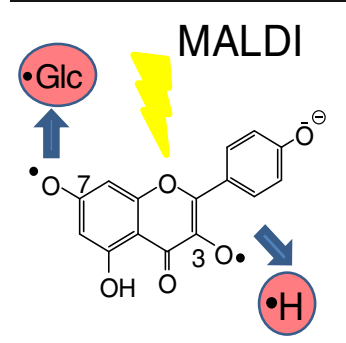

Homolytic cleavage

Abstract. Negative-ion matrix-assisted laser desorption/ionization (MALDI) timeof-flight mass spectra and tandem mass spectra of flavonoid mono- $O$-glycosides showed the irregular signals that were 1 and/or 2 Da smaller than the parent deprotonated molecules $\left([\mathrm{M}-\mathrm{H}]^{-}\right)$and the sugar-unit lost fragment ions ([M Sugar $-\mathrm{H}^{-}$). The 1 and/or $2 \mathrm{Da}$ mass shifts are generated with the removing of a neutral hydrogen radical $\left(\mathrm{H}^{*}\right)$, and/or with the homolytic cleavage of the glycosidic bond, such as $\left[\mathrm{M}-\mathrm{H}^{*}-\mathrm{H}\right]^{-},\left[\mathrm{M}-\mathrm{Sugar}-\mathrm{H}^{*}-\mathrm{H}\right]^{-}$, and $\left[\mathrm{M}-\mathrm{Sugar}-2 \mathrm{H}^{*}-\mathrm{H}\right]^{-}$. It was revealed that the hydrogen radical removes from the phenolic hydroxy groups on the flavonoids, not from the sugar moiety, because the flavonoid backbones themselves absorb the laser. The glycosyl positions depend on the extent of the hydrogen radical removals and that of the homolytic cleavage of the glycosidic bonds. Flavonoid mono-glycoside isomers were distinguished according to their TOF MS and tandem mass spectra. Key words: Flavonoid, Glycoside, Isomeric identification, Homolytic cleavage

Received: 10 June 2013/Revised: 20 September 2013/Accepted: 24 September 2013/Published online: 19 November 2013

\section{Introduction}

$\mathrm{F}^{\mathrm{s}}$ lavonoids are plant secondary metabolites that are stored in glycoside form (Figure 1). In our daily diet, flavonoid glycosides are found in food stuffs (e.g., vegetables, teas, and wines), and have been used for day-to-day health [1, 2] because the flavonoid glycosides have biological activities such as anti-oxidant [3, 4], anti-carcinogenic [5, 6], and antinfluenza virus [7] activities. The hydroxy groups on the aromatic rings of flavonoids have phenolic hydroxy properties, and these chemical properties are thought to cause their biological activities which depending on their glycosyl positions and on the backbone structures. The chemical structures of various flavonoid glycosides have been analyzed by using electrospray ionization (ESI) liquid chromatography mass spectrometry (LC-MS), and combining tandem MS and $\mathrm{MS}^{\mathrm{n}}$ analyses. These techniques are also useful for the differentiation of isomeric flavonoid glycosides [8-12]. Recently, the locations of flavonoid glycosides in plants has been

Electronic supplementary material The online version of this article (doi:10.1007/s13361-013-0764-0) contains supplementary material, which is available to authorized users.

Correspondence to: Tohru Yamagaki ; e-mail: yamagaki@sunbor.or.jp assessed by mass spectral imaging using matrix-assisted laser desorption/ionization mass spectrometry (MALDI-MS) [1316]. MALDI imaging-MS is a potentially powerful tool for the flavonoid analysis in vivo system. Interestingly, phenolic compounds such as dihydroxybenzoic acids showed the unexpected 1 and/or 2 Da mass shifts of the parent deprotonated molecules in the negative-ion MALDI mass spectra [17]. The neutral hydrogen radicals $\left(\mathrm{H}^{*}\right)$ are removed from the phenolic hydroxy groups. In the case of dihydroxybenzoic acids, the deprotonated molecules such as $\left[\mathrm{M}-\mathrm{H}^{*}-\mathrm{H}\right]^{-}$and $\left[\mathrm{M}-2 \mathrm{H}^{*}-\mathrm{H}\right]^{-}$beside $[\mathrm{M}-\mathrm{H}]^{-}$ions were observed in the negative-ion MALDI-MS. The $\left[\mathrm{M}-\mathrm{H}^{*}-\mathrm{H}\right]^{-}$ ion of 2,5-dihydroxybenzoic acid (DHBA) was the most abundant among the isomers. Their relative ion abundances were different among their isomers and they depended on the relative positions of two hydroxy groups and carbohydroxy group [17]. The properties of the phenolic groups of various compounds such as flavonoids should be recognized in MALDI-MS and should be proofed according to their structure. Generally, the mono-isotopic ions of the molecules and fragments are essential for their structure elucidation. The unexpected hydrogen radical removals could hinder correct structure analysis. In the study reported herein, we focused our attention on the MALDI-ionization and fragmentation of flavonoid mono-glycoside isomers. 


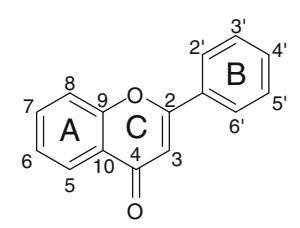

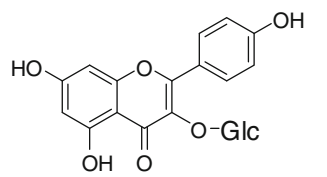

Kaempferol-3-O-GIc<smiles>COc1cc(O)c2c(=O)cc(-c3ccc(OC)c(O)c3)oc2c1</smiles>

Luteolin-4'-O-Glc

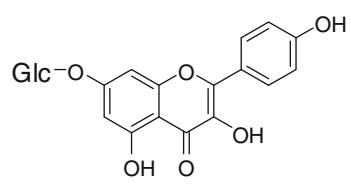

Kaempferol-7-O-GIC

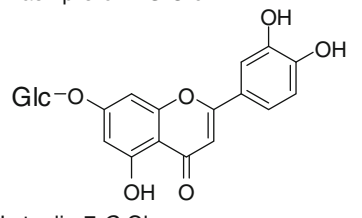

Luteolin-7-O-Glc

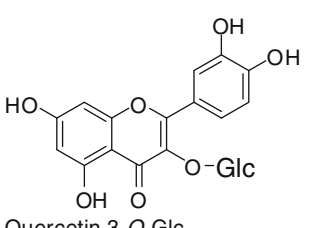

Quercetin-3-O-Glc<smiles>O=c1c(OCCl)c(-c2ccc(O)c(O)c2)oc2cc(O)cc(O)c12</smiles>

Quercetin-3-O-Gal

Figure 1. Structures of flavonoid glycosides. Glc, $\alpha-D-$ glucopyranose; Gal, a-D-galactopyranose; Rhm, $\alpha$-Lrhammopyranose

\section{Experimental}

\section{Samples and Chemicals}

Kaempferol-3-O-glucoside (Kmp-3-Glc), kaempferol-7-Oglucoside (Kmp-7-Glc), luteolin-4'-O-glucoside (Lut-4'$\mathrm{Glc}$ ), luteolin-7-O-glucoside (Lut-7-Glc), quercetin-3-O-glucoside (Qur-3-Glc), quercetin-3-O-galactoside (Qur-3-Gal), and myricetin-3-O-rhamnose (Myr-3-Rhm) were obtained from Extrasynthese, Lyon, France (Figure 1). Analytes were prepared as methanol suspensions $(1 \mathrm{mg} / \mathrm{mL})$. Norharman $(2.5 \mathrm{mg} / \mathrm{mL} 80 \%$ (vol/vol) acetonitrile) served as the matrix. For each spectrum, a $1 \mu \mathrm{L}$ sample containing matrix and analyte was deposited on the ionization platform and then dried.

\section{Mass Spectrometry}

An Ultraflex III MALDI TOF/TOF mass spectrometer (Bruker Daltonics GmbH, Bremen, Germany) was used for all TOF-MS and LID-MS/MS, which is equipped with a $355 \mathrm{~nm}$ wavelength laser generating system (Bruker Smartbeam-II laser). In-source fragmentation was assessed in reflectron-mode TOF-MS. The production spectra were measured in the MS/MS lift mode during post-source decay. Each precursor deprotonated ion $[\mathrm{M}-\mathrm{H}]^{-}$was manually selected at its expected $\mathrm{m} / \mathrm{z}$ value for subsequent laser-induced fragmentation (mass window, $5 \mathrm{Da}$ ). High-resolution mass spectra were acquired using a $9.4 \mathrm{~T}$ Apex FT-ICR mass spectrometer (Bruker Daltonics $\mathrm{GmbH}$ ).

\section{Results and Discussion}

Figure 2 shows the negative-ion reflectron-mode MALDITOF mass spectra of the flavonoid mono- $O$-glycosides Kmp-3-Glc, Kmp-7-Glc, Lut-4'-Glc, and Lut-7-Glc under slightly high irradiation laser power condition, enough to induce in-source fragmentation. All spectra were acquired under the same conditions. In each spectrum (Figure 2, right column), the two most intense peaks are found at $m / z 447$ as the deprotonated molecule $[\mathrm{M}-\mathrm{H}]^{-}$, and at $m / z 285$ as the fragment ion $[\mathrm{M}-\mathrm{Glc}-\mathrm{H}]^{-}$, which arose owing to cleavage of the glycosidic bond. Besides them, 1 and/or 2 Da mass shifts are also present (Figure 2, left and middle columns). The fragment ions would have been generated in MALDI insource. The high-resolution MS spectrum of Kmp-7-Glc (Supplemental Information 1) also contains the peaks at $\mathrm{m} / \mathrm{z}$ 447,446 , and $285,284,283$, which we assigned to the following ions, respectively: $[\mathrm{M}-\mathrm{H}]^{-}\left(\mathrm{m} / z\right.$ 447), $\left[\mathrm{M}-\mathrm{H}^{*}-\right.$ $\mathrm{H}]^{-}\left(m / z\right.$ 446), and $[\mathrm{M}-\mathrm{Glc}-\mathrm{H}]^{-}(\mathrm{m} / z$ 285), [M - Glc $\left.\mathrm{H}^{*}-\mathrm{H}\right]^{-}(m / z 284),\left[\mathrm{M}-\mathrm{Glc}-2 \mathrm{H}^{*}-\mathrm{H}\right]^{-}(m / z 283)$, in which $\mathrm{H}^{*}$ represents a neutral hydrogen radical. The fragment ions including a hydrogen radical $\left(\mathrm{H}^{*}\right)$ are associated with the homolytic cleavages at the phenolic hydroxy groups and glycosidic bonds. Figure 3 shows the fragmentation scheme of Kmp-7-Glc through the heterolytic cleavage and the homolytic cleavage in MALDI-TOF MS and MS/MS. Because the hydrogen radical-removing ions are not commonly found in soft ionization MS of biological compounds such as peptides and sugar chains, we possibly did not assign them correctly if we obtained these peaks of an unknown compound. We could not definitively identify which mono-isotopic ions were associated with the experimental peaks because we did not expect homolytic cleavages. Although monitoring the loss of neutral moieties is a popular means of characterizing glycoside-containing metabolites, irregular fragmentation at a glycosidic bond could hinder the identification of the glycoside substitution. Therefore, for this report, we studied the relationship between hydrogen radical removing and the structures of various flavonoid mono-glycosides. We also studied the mechanism by which the neutral radical removing odd-ions are generated by laser-induced dissociation (LID) in-source and post-source. 

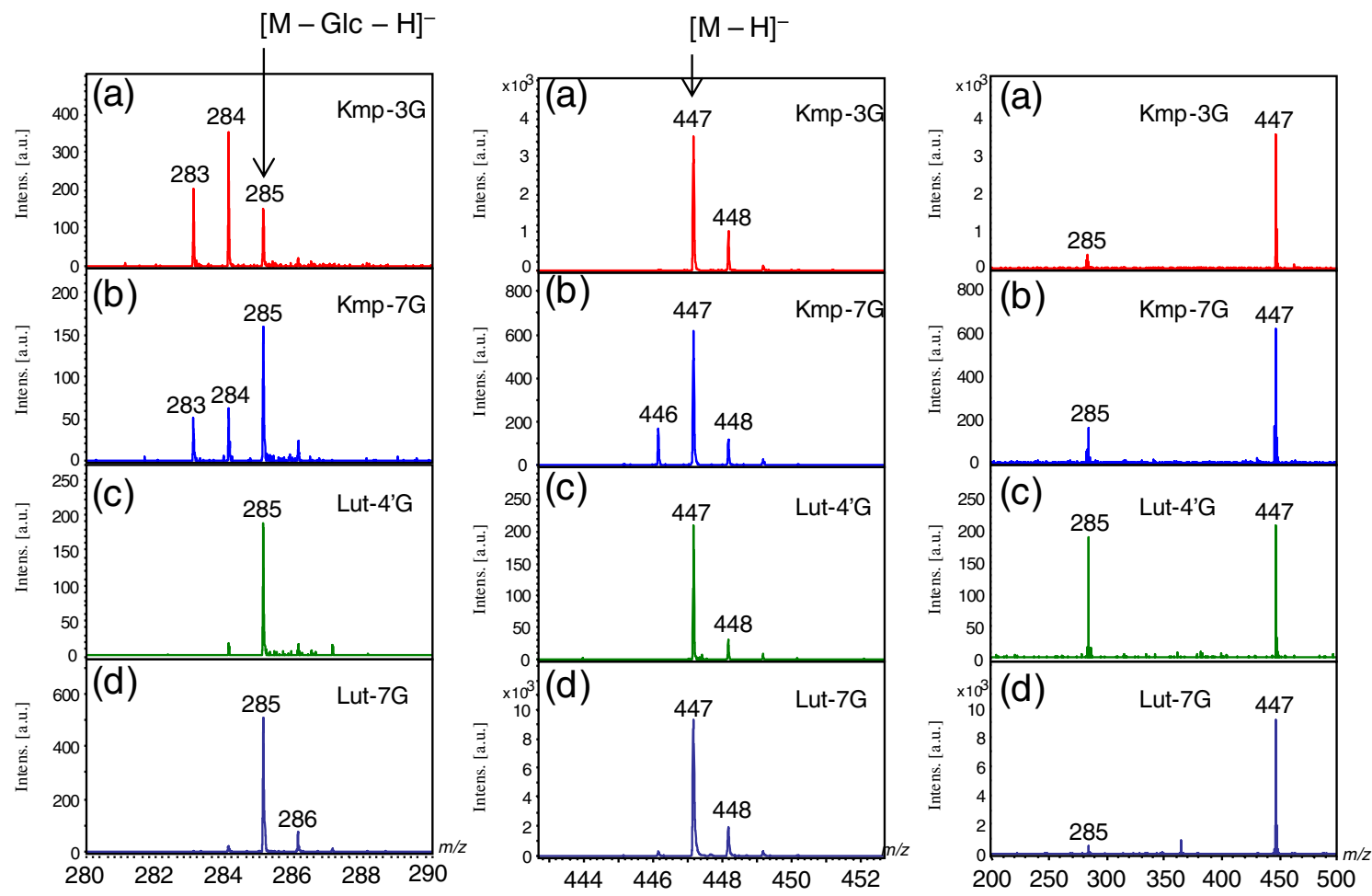

Figure 2. The negative-ion reflectron-mode MALDI-TOF mass spectra of flavonoid glycosides. (a) Kaempferol-3-O-glucoside (Kmp-3-Glc); (b) kaempferol-7-O-glucoside (Kmp-7-Glc); (c) luteolin-4'-O-glucoside (Lut-4'-Glc); (d) luteolin-7-O-glucoside (Lut-7-Glc). The left and middle panels are enlargements around $m / z 285$ and 447 regions, respectively, of the spectra shown on the right

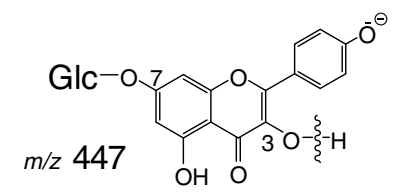

\begin{tabular}{c|c}
$\begin{array}{r}\text { Homolytic } \\
\text { cleavage }\end{array}$ & $-\mathrm{H}^{\bullet}$ \\
$\mathrm{C}-3$
\end{tabular}

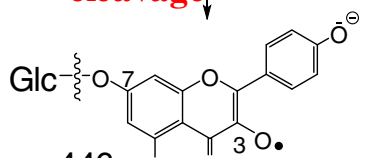

$\mathrm{m} / \mathrm{z} 446$ OH $\mathrm{O}^{3}\left[\mathrm{M}-\mathrm{H}^{\circ}-\mathrm{H}\right]^{-}$

$[\mathrm{M}-\mathrm{H}]^{-}$

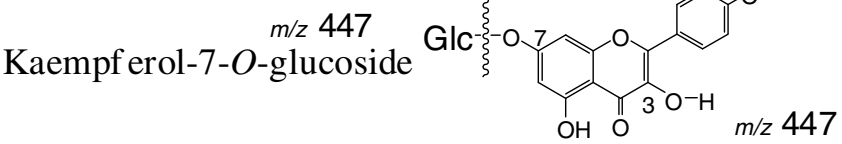

Homolytic - Glc Heterolytic

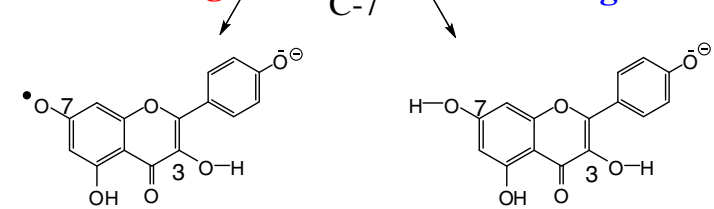

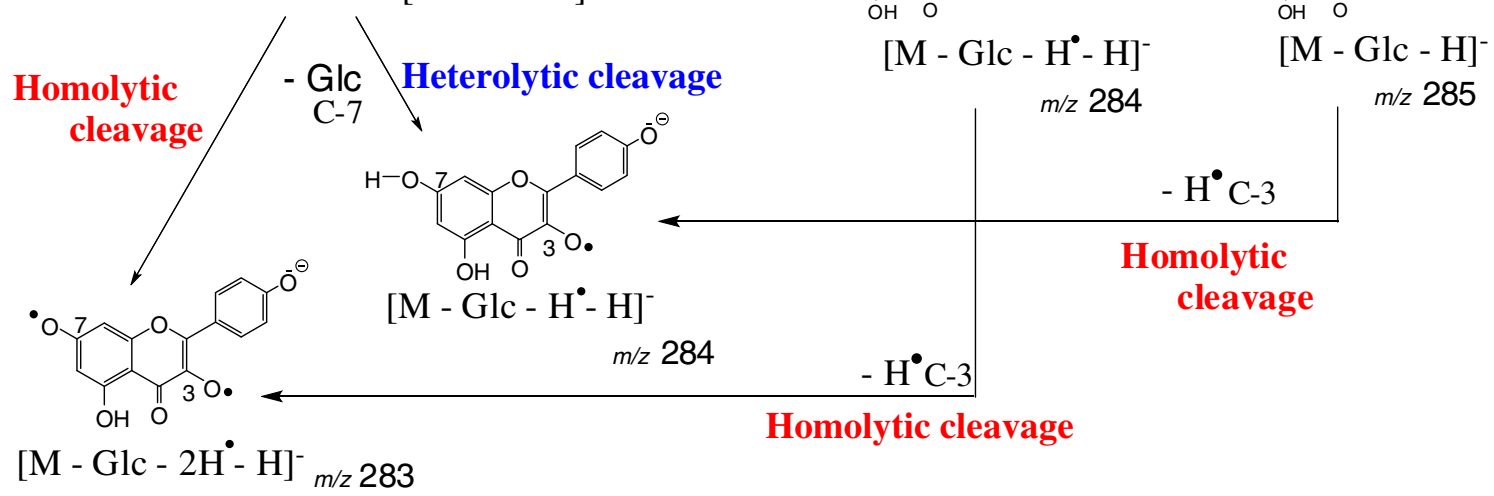

Figure 3. Fragmentation pathway of kaempferol-3-O-glucoside 


\section{Distinguishing Among Isomeric Flavonoid Mono- Glycosides by MALDI-LID MS/MS}

Figure 4 shows the negative-ion MALDI-LID tandem mass spectra of the flavonoid- $\mathrm{O}$-glycoside isomers $\mathrm{Kmp}-3-\mathrm{Glc}$, Kmp-7-Glc, Lut-4'-Glc, and Lut-7-Glc. There are the deprotonated molecule $[\mathrm{M}-\mathrm{H}]^{-}$at $m / z 477$ as the precursor ion and the product ions at $\mathrm{m} / \mathrm{z} 285$ arising from the usual heterolitic cleavage of the glycosyl bond. Interestingly, the relative ion intensity patterns around the ions at $m / z 447$ and 285 differ among the isomers (Figure 4, left and middle column) when the signal intensities are normalized to the intense peaks at $\mathrm{m} / \mathrm{z} 477$ and 285 in the expanded spectra. Thus, they depend on both the sugar positions of the flavonoids and the flavonoid backbone structure. It has been reported that electrospray ionization (ESI) collision-induced dissociation (CID) homolytically cleaved only at glycosidic bonds $[8,9,11,12]$. Conversely, MALDI excitation induced hydrogen radical removal from a phenolic hydroxy group as well as the homolytic cleavage of the glycosidic bond. We then studied how the positions of the sugar substitution in flavonoid glycosides affect the MALDI-LID MS/MS fragmentation patterns.

\section{Flavonol 3-O-Glycosides}

In the MALDI-LID tandem mass spectrum of Kmp-3-Glc, the relative intensity of the peak at $m / z 284\left[\mathrm{M}-\mathrm{Glc}-\mathrm{H}^{*}-\right.$ $\mathrm{H}]^{-}$is the highest among the isomers (Figures 4 and 5), suggesting that the homolytic cleavage is mostly promoted at the 3-O-glycosyl bond of kaempferol. Homolytic cleavage of a flavonol 3-O-glycosidic bond is favored owing to the presence of the 2,3-double bond adjacent to the $\mathrm{C} 4$ carbonyl in ring $\mathrm{C}$ because of the electron conjugated system (Figure 1), which was consistent with the ESI-CID MS/MS analyses [9]. The phenoxy radical is probably stabilized by $\pi$-electron delocalization (Supplemental Information 2).

To determine if the fragmentation of Kmp-3-Glc is a common feature of flavonoid 3-O-glycosides, the spectra of Qrc-3-Glc, Qrc-3-Gal, Myr-3-Gal, and Myr-3-Rhm were acquired (Supplemental Information 3). The abbreviation of Hex means hexose, such as glucose and galactose, and that of sugar means all monosaccharide units including rhamnose. The peaks at $m / z 300\left[\mathrm{M}-\mathrm{Hex}-\mathrm{H}^{*}-\mathrm{H}\right]^{-}$in the tandem mass spectra of Qrc-3-Glc (Supplemental Information 3a) and Qrc-3-Gal (Supplemental Information 3b) are generated by homolytic cleavage of the 3-O-glycosidic bond
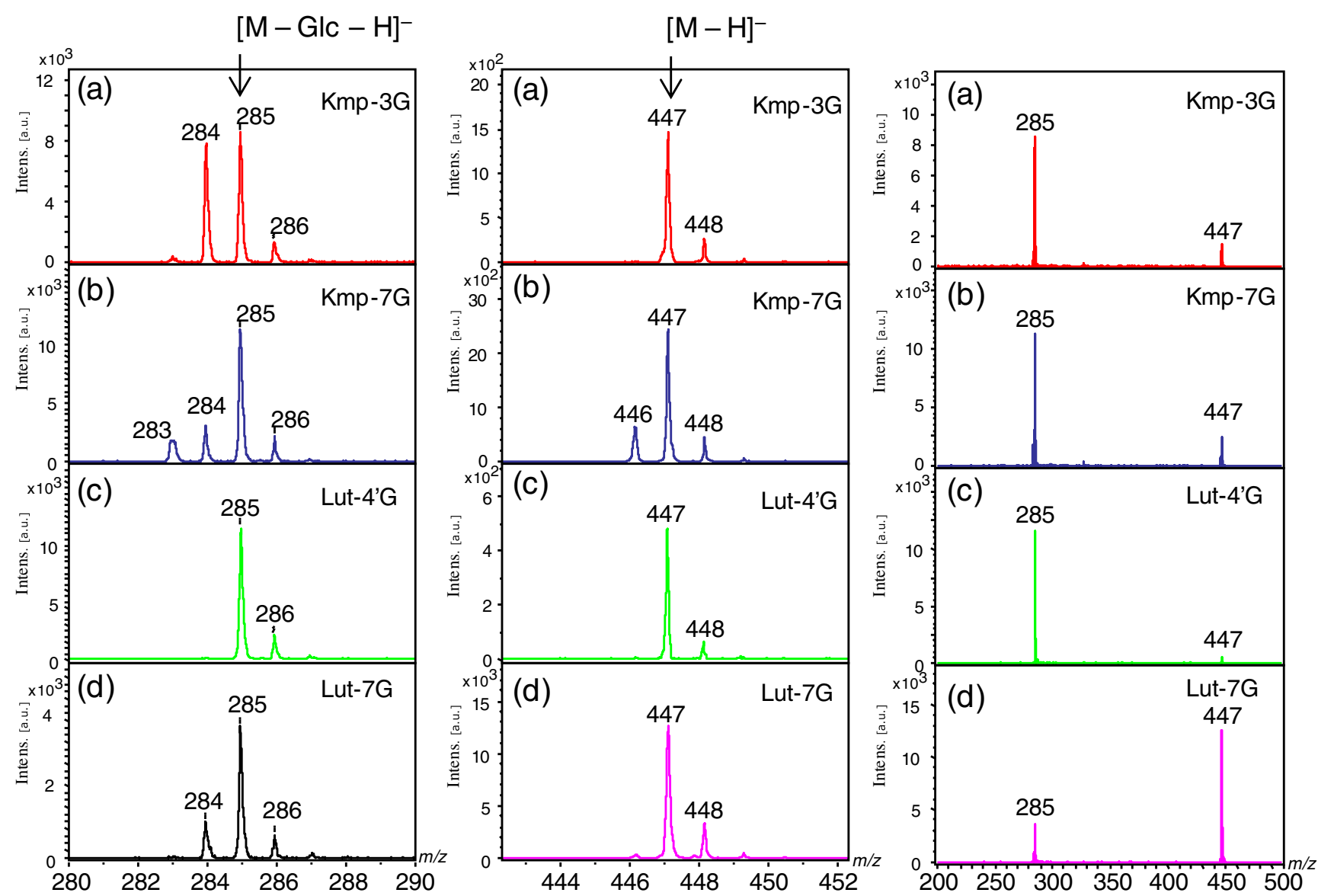

Figure 4. The negative-ion mode MALDI laser-induced dissociation tandem mass spectra of flavonoid glycosides. (a) Kaempferol-3-O-glucoside (Kmp-3-Glc); (b) kaempferol-7-O-glucoside (Kmp-7-Glc); (c) luteolin-4'-O-glucoside (Lut-4'-Glc); (d) luteolin-7-O-glucoside (Lut-7-Glc). The left and middle panels are enlargements around $\mathrm{m} / z 285$ and 447 regions, respectively, of the spectra shown on the right 


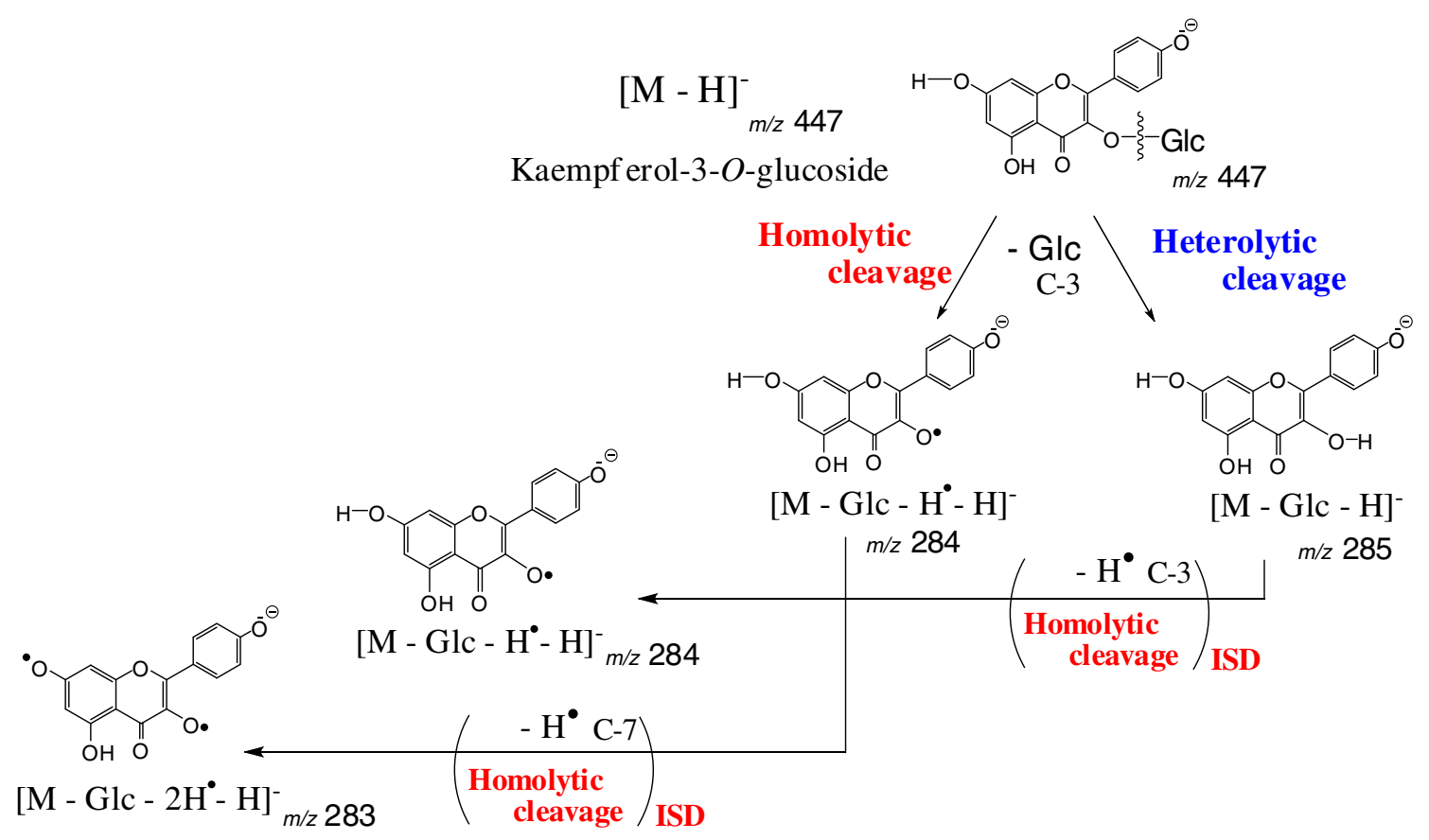

Figure 5. Fragmentation pathway of kaempferol-7-O-glucoside

(Supplemental Information 3, left column). An effect by the different glycosyl moieties glucose and galactose is not apparent. The radical aglycone product ions [M - Sugar $\left.\mathrm{H}^{*}-\mathrm{H}\right]^{-}$of Myr-3-Gal (data not shown) and Myr-3-Rhm (Supplemental Information 3c) also dominates in their product ion spectra. In conclusion, the homolytic cleavage is mostly promoted at the 3-O-glycoside of flavonol in MALDI-LID tandem MS.

The product ions $\left[\mathrm{M}-\mathrm{Hex}-\mathrm{H}^{*}-\mathrm{H}\right]^{-}$and $[\mathrm{M}-\mathrm{Hex}-$ $\mathrm{H}]^{-}$have almost the same intensity among Kmp-3-Glc, Qrc3-Glc, and Qrc-3-Gal (Figure 4 and Supplemental Information 3). In the case of the ESI-MS of flavonoid-3-rutinosides, as the number of hydroxy groups in the B-ring increased, the $\left[\mathrm{M}-\mathrm{Hex}-\mathrm{H}^{*}-\mathrm{H}\right]^{-}$ion peak intensity increased. The extent of the homolytic cleavage at the 3-O-glycosyl bond in MALDI LID differed from that of ESI-CID tandem mass spectra [9]. The relative abundance of the product ion [M Hex $\left.-\mathrm{H}^{*}-\mathrm{H}\right]^{-}$from Myr-3-Gal is greater than that from Qrc3-Gal, which is slightly greater than that from Kmp-3-Glc.

\section{Flavonoid 7-O-Glycosides}

In the MALDI-LID tandem mass spectrum of Kmp-7-Glc, the product ion at $m / z 446\left[\mathrm{M}-\mathrm{H}^{*}-\mathrm{H}\right]^{-}$is observed beside the parent deprotonated molecule at $m / z 477[\mathrm{M}-\mathrm{H}]^{-}$ (Figure 4b). Notably, no such peak is present in the spectra of the flavonoid 3-O-glycosides. Thus, the C-3 hydroxy group (3-OH) in ring $\mathrm{C}$ of $\mathrm{Kmp}-7-\mathrm{Glc}$ is responsible for the hydrogen radical removal from the parent molecule. The C-3 hydroxy group at ring $\mathrm{C}$ of flavonoids is the most active site
[18]. In the previous study, the reactive order of the phenolic hydroxyl groups on quercetin were analyzed with regard to methylation with iodomethane and potassium carbonate in $\mathrm{N}, \mathrm{N}$-dimethyl formamide [18]. The phenolic hydroxy groups were reacted under the order $3-\mathrm{OH}>7-\mathrm{OH}>4^{\prime}-\mathrm{OH}>3^{\prime}-\mathrm{OH}$ [18]. On the other hand, the phenoxy radical at C-3 would be stabilized by $\pi$-electron delocalization (Supplemental Information 4). Flavonoid glycosides absorb UV light at $355 \mathrm{~nm}$ and they get into the high-energy excited states, then, a hydrogen radical can remove preferably at the $\mathrm{C}-3$ phenolic hydroxy group of flavonol. Because the phenolic hydroxy groups on flavonoid glycosides are easily excited by the laser irradiation, it can be assumed that a hydrogen radical is homolytically removed from a hydroxy group in the flavonoid backbone and not from the sugar unit. Notably, the product ion $\left[\mathrm{M}-\mathrm{H}^{*}-\mathrm{H}\right]^{-}$was not detected in ESI-CID tandem mass spectra of flavonoid-7-Oglycosides [3], which suggests that laser irradiation induces hydrogen radical removal from flavonoids.

The tandem mass spectrum of Kmp-7-Glc contains the product ion peaks at $m / z 283\left(\left[\mathrm{M}-\mathrm{Glc}-2 \mathrm{H}^{*}-\mathrm{H}\right]^{-}\right)$and $m / z$ $284\left(\left[\mathrm{M}-\mathrm{Glc}-\mathrm{H}^{*}-\mathrm{H}\right]^{-}\right)$, which are generated by the hydrogen radical removal from the $\mathrm{C}-3$ phenolic hydroxy group and/or by the homolytic cleavage of the 7-O-glycosidic bond (Figures 3 and 4). The product ion at $m / z 284\left[\mathrm{M}-\mathrm{Glc}-\mathrm{H}^{*}-\right.$ $\mathrm{H}]^{-}$are generated by two possible fragment pathways (Figure 3 ) because the product ion of $\left[\mathrm{M}-\mathrm{Glc}-\mathrm{H}^{*}-\mathrm{H}\right]^{-}$of Lut-7-Glc is generated through the homolytic cleavage of the 7-O-glycosidic bond in tandem MS (Figure 4d). Therefore, the homolytic cleavage of the 7-O-glycosidic bond in Kmp-7-Glc also progresses in post-source decay (PSD) (Figure 3). 
The following hypothesis is summarized; the C-3 phenoxy radical is more stable and can be generated more easily than 7-O-phenoxy radical of flavonoids (C-3 radical $>$ C-7 radical) (Figures 2, 3, 4 and 5), and homolytic cleavage of a glycosidic bond between $\mathrm{O}-\mathrm{C}$ occurs more readily than just that of a sigma bond between $\mathrm{O}-\mathrm{H}$ of phenolic hydroxy group with a hydrogen radical removal at the same position. Therefore, the C-3 and C-7 chemical bonds cleave homolytically as the following order:

$$
\begin{aligned}
& \text { 3-O-glycosyl bond }{ }^{\text {(homo) }}>\mathrm{C}-3 \mathrm{O}-\mathrm{H}^{\text {(homo) }} \\
& \text { 7-O-glycosyl bond }
\end{aligned}
$$

Considering the abundant product ions between Kmp-3Glc and Kmp-7-Glc, the ion $\left[\mathrm{M}-\mathrm{Glc}-\mathrm{H}^{*}-\mathrm{H}\right]^{-}$is the major one in the tandem mass spectrum of Kmp-3-Glc because the homolytic cleavage at 3-O-glycosidic bond contributes it mainly; whereas the product ions $[\mathrm{M}-\mathrm{Glc}-$ $\left.\mathrm{H}^{*}-\mathrm{H}\right]^{-}$and $\left[\mathrm{M}-\mathrm{Glc}-2 \mathrm{H}^{*}-\mathrm{H}\right]^{-}$were observed in the tandem mass spectrum of Kmp-7-Glc with low abundance because the contribution are almost the same between the hydrogen radical removal at the $\mathrm{C}-3$ phenolic hydroxy group and the homolytic cleavage at $7-O$-glycosidic bond as the formula (1).

\section{Distinguishing of Lut-7-Glc, Kmp-7-Glc, and Lut- 4'-Glc}

The structure of the unsubstituted C-3 hydroxy group of flavonols inevitably causes a hydrogen radical removal from the molecule in the negative-ion MALDI-MS. The peak at $\mathrm{m} / \mathrm{z} 446$ is abundantly detected beside the parent and precursor ion at $m / z 447$ in the negative-ion MALDI TOF MS and tandem MS spectra of Kmp-7-Glc (Figures 2 and 4). It makes clear the spectral difference between Kmp-7-Glc and Lut-7-Glc. After the 7-O-glycosyl bond cleaves homolytically and the 1 Da-small fragment ions at $\mathrm{m} / \mathrm{z} 284$ are generated, the 2 Da-small fragment ion at $\mathrm{m} / z 283$ is generated only from Kmp-7-Glc because of the unsubstituted C-3 hydroxy group of flavonol (Figures 2 and 4).

The relative intensity of the peak at $\mathrm{m} / \mathrm{z} 285$ to that of $\mathrm{m} / \mathrm{z}$ 447 in the Lut-7-Glc tandem MS spectrum is much less intense in the spectrum of Kmp-7-Glc (Figure 4). The aglycone ion at $\mathrm{m} / \mathrm{z} 285$ from Kmp-7-Glc is much more easily generated than that of Lut-7-Glc. Therefore, Lut-7-Glc and Kmp-7-Glc can be identified by their MALDI-TOF and tandem mass spectra.

When comparing the tandem MS spectra of Lut-4'-Glc and Lut-7-Glc (Figure 4), the product ion at $m / z 284$ is found only in the tandem mass spectrum of Lut-7-Glc. Conversely, the cleavage of the glycosyl bond in ring $\mathrm{B}$ does not occur homolytically in Lut-4'-Glc. The 4'-O-glycosyl bond in B ring of Lut-4'-Glc cleaves more easily than that in A ring of Lut-7-Glc totally. Consequently, all of Lut-7-Glc, Kmp-7Glc, and Lut-4'-Glc are distinguished by using their mass spectra.

\section{In-Source Decay Versus Post-Source Decay}

Comparing the reflectron-mode MALDI-TOF MS spectra and the LID tandem MS spectra (Figures 2 and 4), we can discuss in-source and post-source decay product ions. The fragment ion $\left[\mathrm{M}-\mathrm{Glc}-2 \mathrm{H}^{*}-\mathrm{H}\right]^{-}$at $m / z 283$ of Kmp-3Glc is observed in the reflectron-mode MALDI-TOF MS spectrum (Figure 2a), but it is not observed in the MALDILID tandem MS spectrum (Figure 4a). Thus, the ion [M Glc $\left.-2 \mathrm{H}^{*}-\mathrm{H}\right]^{-}$at $m / z 283$ of $\mathrm{Kmp}-3$-Glc is produced from in-source decay (ISD), but not from PSD process. Laserinduced ISD effectively induces phenoxy radicals because the flavonoid moiety is excitable in MALDI process. Because the ion peak $\left[\mathrm{M}-\mathrm{Glc}-2 \mathrm{H}^{*}-\mathrm{H}\right]^{-}$at $m / z 283$ in the mass spectrum of Kmp-3-Glc is as sharp as the other peaks in that spectrum (Figure 2a), it is suggested to be generated on the same time scale as the laser pulses. Conversely, the tandem mass spectrum of Kmp-3-Glc hardly includes the product ion of $\left[\mathrm{M}-\mathrm{Glc}-2 \mathrm{H}^{*}-\mathrm{H}\right]^{-}$at $m / z 283$ (Figure 4a). The glycosyl bond cleavages are proceeded both heterolytically as [M - Glc $-\mathrm{H}]^{-}(\mathrm{m} / \mathrm{z} 285)$ and homolytically as $\left[\mathrm{M}-\mathrm{Glc}-\mathrm{H}^{*}-\mathrm{H}\right]^{-}(\mathrm{m} / \mathrm{z} 284)$ in post-source decay. The metastable ion decayed within nanoseconds during the second TOF step. During the PSD process, the Kmp-3-Glc ion $\left[\mathrm{M}-\mathrm{Glc}-\mathrm{H}^{*}-\mathrm{H}\right]^{-}$ at $\mathrm{m} / \mathrm{z} 284$ is produced mainly via homolytic glycosidic bond cleavage rather than via the further hydrogen radical removal from the species $[\mathrm{M}-\mathrm{Glc}-\mathrm{H}]]^{-}(\mathrm{m} / \mathrm{z} 285)$ (Figures $4 \mathrm{a}$ and 5$)$. Hydrogen radical removal at a phenolic hydroxy group via homolytic cleavage occurs through the process of ISD process rather than that of PSD.

The spectra of Lut-7-Glc differ between the reflectronmode TOF MS and tandem MS. The product ion at $\mathrm{m} / \mathrm{z} 284$ of Lut-7-Glc is generated in the post-source decay process (Figure 4d), in contrast, the ion is hardly generated in insource decay process (Figure 2d). The hydroxy radical hardly removes from the B-ring phenolic hydroxy groups because of the ring system, and from the $\mathrm{C}-5$ phenolic hydroxy group because of the hydrogen bonding to the C-4 carbonyl group. Only the homolytic cleavage is induced with 7-O-glycosyl bond cleavage.

The Kmp-7-Glc product ion $\left[\mathrm{M}-\mathrm{Glc}-2 \mathrm{H}^{*}-\mathrm{H}\right]^{-}$at $\mathrm{m} / \mathrm{z}$ 283 is generated by ISD and PSD processes; however, the peak widths differs. The peak at $m / z 283$ is as sharp as the peaks at $\mathrm{m} / \mathrm{z} 284$ and 285 in the ISD mass spectrum (Figure 2b). The peak at $\mathrm{m} / z 283$ in the LID tandem mass spectrum is broader than the peaks at $\mathrm{m} / \mathrm{z} 284$ and 285 , suggesting that the ion peak at $\mathrm{m} / \mathrm{z} 283$ is also generated by PSD process via the hydrogen radical removing at the C-3 phenolic hydroxy group of Kmp-7-Glc. It was because the homolytic cleavage of the C-3 hydroxy group preferred to the others as formula (1). 


\section{Conclusions}

Homolytic cleavage at phenolic hydroxy group $(\mathrm{O}-\mathrm{H})$ instead of a sugar hydroxy group is favored when the neutral hydrogen radical is removed. The flavonoid radical would be stabilized by $\pi$-electron delocalization. We previously found that the removal of a neutral hydrogen radical from dihydroxybenzoic acids depends on the positions of the two hydroxy groups with respect to the carboxylic acid [17]. The hydrogen radical removes most frequently from the phenolic hydroxy group of 2,5dihydroxybenzoic acid among six isomers. In the case of flavonoid glycosides, the glycosyl positions also influence the extent to the hydrogen radical removals. The hydrogen radical is released from the C-3 hydroxy group in flavonoids more easily than the other hydroxy groups as summarized in the formula (1). The hydrogen radical removal at the C-3 phenolic hydroxy group of Kmp-7-Glc occurs both in ISD and PSD, and that of the C-7 phenolic hydroxy group of Kmp-3-Glc occurs in ISD but not in PSD. It is indicated that the C-3 phenoxy radical is more stable and it can be generated more easily than the C-7 phenoxy radical of flavonoids (Supporting Information 2 and 4).

Homolytic cleavage of a glycosidic bond occurs more readily than that of a sigma bond of phenolic hydroxy group with a hydrogen radical removal as shown in the formula (1). Those spectral patterns of the flavonoid mono-glycosides can identify their structures. A detailed understanding of how hydrogen radicals are removed from polyphenolic compounds is essential for their unambiguous identification and for semiquantitative analyses. In this study, we found that the homolytic cleavage of flavonoid phenolic hydroxy group occurs and that positional isomers could be distinguished by negative-ion reflectron-mode MALDI-MS and LID-MS/MS because of the positional specificity of hydrogen radical removal.

\section{Open Access}

This article is distributed under the terms of the Creative Commons Attribution License which permits any use, distribution, and reproduction in any medium, provided the original author(s) and the source are credited.

\section{References}

1. Middleton Jr., E., Kandaswami, C., Theoharides, T.C.: The effects of plant flavonoids on mammalian cells: implications for inflammation, heart disease, and cancer. Pharmacol Rev 52, 673-751 (2000)
2. Williamson, G., Manach, C.: Bioavailability and bioefficacy of polyphenols in humans. II. Review of 93 intervention studies. Am J Clin Nutr 81(Suppl), 243S-255S (2005)

3. Heim, K.E., Tagliaferro, A.R., Bobilya, D.J.: Flavonoid antioxidants: chemistry, metabolism, and structure-activity relationships. J Nutr Biochem 13, 572-584 (2002)

4. Lopez-Lopez, G., Moreno, L., Cogolludo, A., Galisteo, M., Ibarra, M., Duarte, J., Lodi, F., Tamargo, J., Perez-Vizcaino, F.: Nitric oxide (NO) scavenging and NO protecting effects of quercetin and their biological significance in vascular smooth muscle. Mol. Phamacol. 65, 851-859 (2004)

5. Lu, J., Papp, L.V., Fang, J., Rodriguez-Nieto, S., Zhivotovsky, B., Holmgren, A.: Inhibition of Mammalian thioredoxin reductase by some flavonoids: implications for myricetin and quercetin anticancer activity. Cancer Res 66, 4410-4441 (2006)

6. Brusselmans, K., Vrolix, R., Verthoeven, G., Swinnen, J.V.: Induction of cancer cell apoptosis by flavonoids is associated with their ability to inhibit fatty acid synthase activity. J Biol Chem 280, 5636-5645 (2005)

7. Svov, V.M., Galabov, A.S., Tantcheva, L.P., Mileva, M.M., Pavlova, E.L., Stoeva, E.S., Braykova, A.A.: Effects of rutin and quercetin on monooxygenase activities in experimental influenza virus infection. Exp Toxicol Pathol 58, 59-64 (2006)

8. Vukics, V., Guttman, A.: Structural characterization of flavonoid glycosides by multi-stage mass spectrometry. Mass Spectrom Rev 29, $1-16(2010)$

9. Hvattum, H., Ekeberg, D.: Study of the collision-induced radical cleavage of flavonoid glycosides using negative electrospray ionization tandem quadrupole mass spectrometry. J Mass Spectrom 38, 43-49 (2003)

10. Davis, B.D., Brodbelt, J.S.: Determination of the glycosylation site of flavonoid monoglucosides by metal complexation and tandem mass spectrometry. J Am Soc Mass Spectrom 15, 1287-1299 (2004)

11. Cuyckens, F., Claeys, M.: Determination of the glycosylation site in flavonoid mono- $\mathrm{O}$-glycosides by collision-induced dissociation of electrospray-generated deprotonated and sodiated molecules. J Mass Spectrom 40, 364-372 (2005)

12. Cuyckens, F., Claeys, M.: Mass spectrometry in the structural analysis of flavonoids. J Mass Spectrom 39, 1-15 (2004)

13. Zhang, H., Cha, S., Yeung, E.S.: Colloidal graphite-assisted laser desorption/ionization $\mathrm{MS}$ and $\mathrm{MS}^{\mathrm{n}}$ of small molecules. 2. Direct profiling and MS imaging of small metabolites from fruits. Anal Chem 79, 6575-6584 (2007)

14. Cha, S., Zhang, H., Ilarslan, H.I., Wurtele, E.S., Brachova, L., Nikolau, B.J., Yeung, E.S.: Direct profiling and imaging of plant metabolites in intact tissues by using colloidal graphite-assisted laser desorption ionization mass spectrometry. Plant J 55, 348-360 (2008)

15. Yoshimura, Y., Enomoto, H., Moriyama, T., Kawamura, Y., Setou, M., Zaima, N.: Visualization of anthocyanin species in rabbit eye blueberry Vaccinium ashei by matrix-assisted laser desorption/ ionization imaging mass spectrometry. Anal Bioanal Chem 403, 1885-1895 (2012)

16. Yoshimura, Y., Zaima, N., Moriyama, T., Kawamura, Y.: Different localization patterns of anthocyanin species in the pericarp of black rice revealed by imaging mass spectrometry. PLoS One 7, e31285 (2012)

17. Yamagaki, T., Watanabe, T.: Hydrogen radical removal causes complex overlapping isotope patterns of aromatic carboxylic acids in negativeion matrix-assisted laser desorption/ionization mass spectrometry. Mass Spectrom. 1, A0005 (2012)

18. Suzuki, M., Kimura, T., Yamagishi, K., Shinmoto, H., Yamaki, K.: Characterization of quercetin hydroxy group. (In Japanese). Tohoku Agric. Res 56, 259-260 (2003) 